Schmerz 2012 $26: 121-122$

DOI 10.1007/s00482-012-1162-4

(c) Deutsche Gesellschaft zum Studium des Schmerzes. Published by Springer-Verlag all rights reserved 2012

\section{F. Petzke}

Schmerzklinik im Zentrum Anästhesiologie, Rettungs- und Intensivmedizin, Universitätsmedizin Göttingen

\section{Neue Einsichten und Entscheidungshilfen aus Verbraucherberichten?}

Die Behandlungsoptionen des Fibromyalgiesyndroms (FMS) sind vielfältig und in Bezug auf den klinischen Einsatz und Nachweis ihrer Wirksamkeit sehr heterogen. Trotz relevanter Fortschritte können meist keine großen und lang anhaltenden positiven Effekte erzielt werden - Patienten werden deshalb im Verlauf vielfältige Behandlungen wahrnehmen [1]. Entsprechend ähnelt die alltägliche Versorgungssituation der Patienten einem großen Gemischtwarenladen mit Angeboten unterschiedlicher Qualität, erbracht von einem Spektrum seriöser bis suspekter Anbieter. Wie findet man sich hier als Verbraucher, also als Patient, aber auch als unabhängiger Verordner und Berater, d. h. als Arzt, zurecht? In klassischen randomisierten Studien wird die Wirksamkeit von Behandlungen unter Idealbedingungen („efficacy“) untersucht, Ziel ist eine hohe interne Validität - zumindest sollten die Studien so ideal sein, dass die Wirksamkeit auch nachgewiesen werden kann. Oft findet man in diesem Kontext die konkrete Situation und die Bedürfnisse eines spezifischen Patienten nicht wieder, der vielleicht zu alt ist, das falsche Geschlecht hat oder zusätzliche Komorbiditäten aufweist - die externe Validität der Ergebnisse ist eingeschränkt. Auch werden viele Therapien mangels Interesse gar nicht erst erforscht, andere wegen akademischer oder finanzieller Interessen im Übermaß untersucht.

\section{Welche Auswahlkriterien?}

Auf welcher Grundlage kann aus verschiedenen wirksamen Therapien die am besten geeignete ausgewählt werden? Studien, die ähnliche Therapien direkt vergleichen, sind nicht nur in Bezug auf das FMS rar, sowohl zu medikamentösen als auch zu anderen Behandlungen. Vergleiche zwischen unterschiedlichen therapeutischen Modalitäten sind noch seltener. Neben der uneinheitlichen externen Validität schränken Unterschiede in der Methodik und den gewählten Erfolgsparametern auch die indirekte Vergleichbarkeit der Ergebnisse ein. Metaanalysen und systematische Übersichten basieren in ihrer Methodik insbesondere auf Studien definierter Qualität und fassen durch eine standardisierte Studienanalyse diese Ergebnisse zusammen. Bei der Erstellung von Leitlinien werden durch einen transparenten Prozess auf Basis dieser (eingeschränkten) wissenschaftlichen Erkenntnisse praktisch umsetzbare Empfehlungen für Patient und behandelnden Arzt im klinischen Alltag formuliert. Die S3Leitlinie zum FMS wird aktuell auf dieser Grundlage überarbeitet [2].

\section{Was ist Effectiveness-Forschung?}

Gibt es noch andere Wege zu einer realistischen Einschätzung der Wirksamkeit unter Alltagsbedingungen („effective- ness")? Dieser Frage widmet sich der relativ neue Ansatz der Effectiveness-Forschung. Neben pragmatischen und meist vergleichenden klinischen Studien, deren Fokus es ist, für den klinischen Alltag relevante Fragen zu beantworten [3], kommen hier auch Methoden wie die Einschätzung von Nutzen und Risiken therapeutischer Verfahren durch Patienten zum Einsatz - sog. Verbraucherberichte. Häuser et al. [4] präsentieren in dieser Ausgabe von Der Schmerz die Ergebnisse einer aktuellen Befragung von $1661 \mathrm{~Pa}$ tienten mit FMS. Die Patienten wurden überwiegend über Selbsthilfegruppen rekrutiert (1157 Patienten der Deutschen Fibromyalgie-Vereinigung und Deutschen Rheuma-Liga), der Rest überwiegend aus Zentren der Sekundärversorgung. Eine ausführliche Liste möglicher Behandlungen, die auf der S3-Leitlinie basierte, konnte von den Patienten beliebig ergänzt werden. Die individuellen Behandlungserfahrungen (nie, aktuell oder früher) wurden erfasst und auf ihren Nutzen oder Schaden hin bewertet.

Die Ergebnisse sprechen für die Vernunft der befragten Patienten und für eine grundsätzliche Offenheit für alle Behandlungsansätze. Nach der aktuellen Häufigkeit und Nutzenbewertung stehen an erster Stelle simple Dinge der selbstgesteuerten Alltagsbewältigung wie Ablenkung, Ausruhen, körperliche Aktivität und die Eigenanwendung von Wärme. Medika- 
menten wird dagegen der höchste Schaden zugeordnet. In der S3-Leitlinie stark empfohlene Therapien wie aerobes Training, Balneotherapie und kognitive Verhaltenstherapie werden, ebenso wie multimodale Therapien, günstig bewertet (hoher Nutzen bei geringem Schaden). Zusammengefasst unterstützen die Ergebnisse das schrittweise und abgestufte Vorgehen bei der Steigerung der Behandlung, das in der Leitlinie vorgeschlagen wird.

Es gibt aber auch einige wichtige Widersprüche zur Leitlinie. Verfahren der Komplementär- und Alternativmedizin und passive physiotherapeutische Verfahren wie Massagen werden oft positiv eingeschätzt - beide ohne relevanten Schaden. Medikamente werden trotz des hohen zugeordneten Schadens von $81,4 \%$ der Patienten eingenommen, 56\% nehmen Analgetika, 18,5\% Muskelrelaxanzien und $26 \%$ schwache oder starke Opioide. Hypnotika wurden mit dem höchsten Nutzen und geringsten Schaden aller Medikamente bewertet. Alle diese Medikamente werden in der S3-Leitlinie für die Behandlung nicht empfohlen. Von den empfohlenen Medikamenten werden zwar Antidepressiva von $46,4 \%$ und Pregabalin von 7,6\% der Befragten eingenommen, der Nutzen wird aber gering eingeschätzt.

\section{Defizite und Widersprüche}

Helfen diese Ergebnisse bei individuellen Therapieentscheidungen weiter und verraten sie uns etwas über die „effectiveness"? Nach meiner Einschätzung noch nicht viel, auch wenn sie die alltäglichen Erfahrungen gut widerspiegeln und die schwierige und nicht selten hilflose Situation der Patienten verdeutlichen - viele Medikamente und Verfahren wurden trotz eines geringen Nutzens und bewussten Schadenspotenzials häufig eingesetzt. Was fehlt, ist der Kontext der Erkrankungsschwere. Zudem werden mehr Informationen über Subgruppen benötigt, die sich hinter der Datenvielfalt verstecken, so wäre es beispielsweise interessant $z u$ wissen, welche Behandlungen von wem kombiniert werden und ob es eine typische Eskalation gibt. Die Art der Befragung führte zu vielen fehlenden Angaben, was gerade den Vergleich der Verfahren erschwert. Trotz die- ser Einschränkungen zeigen die Ergebnisse das Potenzial solcher Erhebungen auf. Weitergehende Analysen der Daten in der genannten Richtung und eine darauf aufbauende Weiterentwicklung der Methodik sind zu erhoffen.

\section{$>$ Was die Befragung auch deutlich macht, sind klare Diskrepanzen zwischen Evidenz und Patientenbeurteilung.}

Sie gibt damit wichtige Hinweise für relevante Forschungsfragen. Wissen wir beispielsweise schon genug über die Rolle von Opioiden und Analgetika beim FMS? Wem nutzen und wem schaden diese Medikamente? Haben passive physiotherapeutische Verfahren doch einen Stellenwert? Welche Verfahren der Komplementär- und Alternativmedizin sollten weiter evaluiert werden? Oder wird lediglich eine bessere Aufklärung der Patienten über Nutzen und Schaden von Behandlungen benötigt?

Studien wie die von Häuser et al. [4] liefern wichtige Daten, um die Relevanz der wissenschaftlichen und klinischen Evidenz, die in Leitlinien formuliert wird, an der Realität der Patienten und damit der tatsächlichen klinischen Versorgungssituation zu prüfen. Aus diesem Dialog müssen Prioritäten für zukünftige Forschungsaktivitäten entwickelt werden, die letztlich die Wahl der Methodik bestimmen (Fokus auf „efficacy“ vs. „effectiveness"). Patienten mit chronischen Schmerzen und ihre Ärzte können aus der Einschätzung von Behandlungen in Bezug auf Nutzen und Risiken viel voneinander lernen; gerade dieser Prozess kann eine Kommunikation auf Augenhöhe ermöglichen und mittelfristig helfen, die Angebote im Gemischtwarenladen zu sortieren und auf das Wesentliche zu reduzieren.

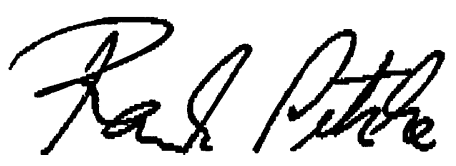

F. Petzke

\section{Korrespondenzadresse}

\section{PD Dr. F. Petzke}

Schmerzklinik im Zentrum Anästhesiologie, Rettungs- und Intensivmedizin, Universitätsmedizin Göttingen Robert-Koch-Str. 40, 37075 Göttingen frank.petzke@med.uni-goettingen.de

Interessenkonflikt. Der korrespondierende Autor weist auf folgende Beziehungen hin: Beratertätigkeit für Grünenthal, Janssen-Cilag; Tätigkeit als Referent für und Reisekostenerstattung durch Eli Lilly.

\section{Literatur}

1. Marschall U, Arnold B, Häuser W (2012) Behandlung und Krankheitskosten des Fibromyalgiesyndroms in Deutschland - eine Analyse der Daten der Barmer Ersatzkasse des Jahres 2008-2009. Schmerz 25:402-410

2. AWMF. http://www.awmf.org/leitlinien/detail/ II/041-004.html. Zugegriffen: 14. März 2012

3. Witt CM, Manheimer $E$, Hammerschlag R et al (2012) How well do randomized trials inform decision making: systematic review using comparative effectiveness research measures on acupuncture for back pain. PLOS One 7:e32399

4. Häuser W, Jung E, Erbslöh-Möller B et al (2012) Der deutsche Fibromyalgieverbraucherbericht. Nutzen und Schaden von Behandlungen des Fibromyalgiesyndroms. Schmerz 26. DOI 10.1007/s00482012-1161-5 